\title{
Postural hypotension in a patient with acute myelitis
}

\author{
J Kalita, UK Misra
}

\begin{abstract}
Summary
A case of acute transverse myelitis with severe postural hypotension is described.
\end{abstract}

Keywords: hypotension, myelitis

Acute transverse myelitis is an acute loss of sensory, motor and bladder functions without evidence of antecedent neurologic disease or spinal cord compression. ${ }^{1}$ Autonomic dysfunction in acute myelitis is common and manifests with bowel, bladder and sexual dysfunction, although postural hypotension is extremely rare.

\section{Case report}

A 43-year-old man was admitted with a history of acute onset of lower limb weakness which reached its peak within 48 hours. He also noted impaired sensations below the midthoracic region, retention of urine and faecal incontinence. On the tenth day of his illness, he had a syncopal attack when he tried to sit up. There was no history of preceding fever, vaccination, sexually transmitted diseases, tuberculosis or a similar illness in the past.

The patient was of average build and nutrition. His neurological examination revealed wasting of both legs, with flaccid, areflexic paraplegia (grade 0 on the $0-5$ scale of the Medical Research Council). Truncal power was below $60 \%$ but the upper limbs were normal. Pinprick and vibration sensations were reduced below the sixth thoracic spinal level and the joint position sensations were absent in both lower limbs. There was no vertebral tenderness or deformity.

Autonomic function tests: the pulse was 82 beats/min and regular. After recording the resting blood pressure, which was $100 / 64 \mathrm{mmHg}$, the patient was made to sit up with his legs hanging down from the bed; immediately after sitting his blood pressure fell to $62 / 36 \mathrm{mmHg}$ and he developed syncope. In a supine position the patient was asked to clinch his fist for $5 \mathrm{~min}$ following which his blood pressure did not reveal any change. After putting his left arm in ice cold water for one minute the blood pressure in the right arm was $100 / 68 \mathrm{mmHg}$. Electrocardiogram (ECG) did not reveal sinus arrhythmia. On deep breathing (six/min) the ratio of the $R R$ interval during expiration and inspiration was 0.8 (normal $\geqslant 1.2$ ). The $R R$ ratio during valsalva manoeuver was calculated by asking the patient to blow to raise the mercury column by $40 \mathrm{~mm}$ for $10 \mathrm{~s}$ with ongoing ECG recording. The ratio of the longest RR interval after the strain and the shortest $R R$ interval during the strain was 0.9 (normal $\geqslant 1.4$ ). Schimer's test was negative and salivation was normal. Pupils were $3 \mathrm{~mm}$, symmetrical and reacted normally to light and accommodation. The patient's skin was dry and scaly below the waist and there was no sweating in the groin and lower limbs after keeping him in a warm environment for $30 \mathrm{~min}$, although there was sweating on face, axilla and palm. The patient had faecal incontinence and retention of urine although urodynamic studies were not possible.

His haemoglobin, blood counts and urinalysis were normal. Erythrocyte sedimentation rate was $32 \mathrm{~mm}$ for the first hour. Blood urea, serum creatinine, blood sugar and liver function tests were normal. Antinuclear antibodies, rheumatoid factor, anti-dsDNA and VDRL were negative. Cerebrospinal fluid revealed $60 \mathrm{mg} / \mathrm{dl}$ protein, $48 \mathrm{mg} / \mathrm{dl}$ sugar, and 7 lymphocytes $/ \mathrm{mm}^{3}$ without any acid-fast bacilli, or fungi. Spinal magnetic resonance imaging (MRI) revealed diffuse hyperintense lesions on $T_{2}$ in the whole of the thoracic spinal cord and hypointensity on $T_{2}$ in the conus medullaris. A few areas of hyperintensity were seen in all the sequences in the thoracic cord which was suggestive of haemorrhage (figure). The vertebral bodies were normal. Motor pathways to the lower limbs were inexcitable on both cortical and spinal stimulation. Central motor conduction time to the upper limbs was normal. Tibial somatosensory-evoked potentials (SEPs) were unrecordable bilaterally but median SEPs were normal. Nerve conduction studies revealed unrecordable peroneal conduction due to severe neurogenic wasting, but sural sensory nerve conduction velocity was normal $(43.9 \mathrm{~m} / \mathrm{s}, 19.0 \mu \mathrm{V})$. Concentric needle electromyograms revealed fibrillations and sharp waves in vastus medialis, tibialis anterior, gastrocnemius, extensor digitorum brevis and lumbar paraspinal muscles. Motor unit potentials were unrecordable from the lower limb muscles. Electromyogram of the abductor pollicis brevis, biceps, and branchioradialis were normal. The patient was managed by intermittent catheterisation, bulk laxatives, indomethacin $50 \mathrm{mg}$ bid, elastic leg bandage and physiotherapy, including postural training.

He was discharged after 15 days of hospital
Misra

Accepted 28 June 1995 


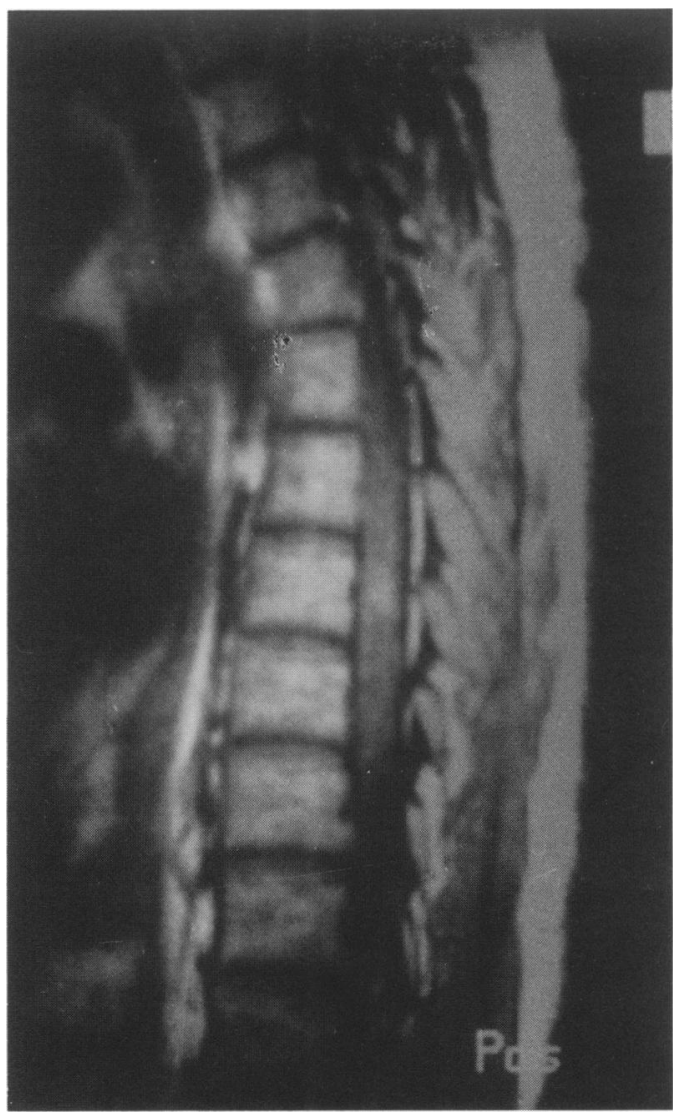

Figure Spinal MRI, $T_{1}$ sequence showing hyperintense lesion in the dorsal region

stay. Three months later he was able to sit without any symptoms of postural hypotension; there was no improvement in motor or sensory symptoms and he still needed intermittent self-catheterisation.

\section{Discussion}

Our patient with acute myelitis had an unusual manifestation of severe postural hypotension leading to disabling syncopal attacks which interfered with his rehabilitation. Autonomic control of blood pressure is complex, being an interplay between parasympathetic and sympathetic drive. Vagal stimulation produces vasodilatation and bradycardia leading to hypotension which is normally counteracted by sympathetic drive, producing tachycardia and vasoconstriction. $^{2}$ The causes of postural hypotension are given in box 1 .

In our patient the extensive spinal cord damage was evidenced by anterior horn cells loss (muscle wasting and electromyogram changes), posterior column (absent joint position sense and tibial SEPs) and spinothalamic involvement (loss of pinprick sensation below sixth thoracic spinal level). The corticospinal dysfunction could not be documented because of severe anterior horn cell loss. These findings suggest both grey and white matter involvement. It is likely that intermediolateral horn cells and descending autonomic pathways were also damaged which accounts for severe dysautonomia in this patient. The involvement of sympathetic outflow in our patient was

\section{Causes of postural hypotension}

Primary

- pure autonomic failure

- multisystem atrophy (Shy-Drager syndrome, parkinsonism, olivopontocerebellar atrophy, striatonigral degeneration)

- acute or subacute dysautonomia

\section{Secondary}

- central: hypothalamic and midbrain tumours, multiple sclerosis, Wernicke's encephalopathy, syringobulbia, age-related changes, spinal injury, syringomyelia, spinal tumours, myelitis

- peripheral: afferent (Guillain Barré syndrome, tabes dorsalis, Holmes-Adie syndrome); efferent (diabetes, amyloidosis, porphyria, familial dysautonomia, carcinomatus neuropathy); miscellaneous (autoimmune and collagen disorders, Eaton Lambert syndrome, AIDS, renal failure, Chaga's disease, polyganglionoradiculopathy, familial hyperbradykininism, dopamine $B$ hydroxylase deficiency)

- neurally mediated syncope: vasovagal and micturition syncope, carotid sinus hypersensitivity, glossopharyngeal neuralgia

Box 1

evidenced by lack of rise of blood pressure on cold immersion and handgrip. Spinal MRI revealed signal changes extending from conus medullaries to the first thoracic level with areas of haemorrhage. Bowel and bladder involvement are common in segmental involvement of spinal cord but postural hypotension occurs only in extensive lesions reaching above the fifth thoracic segment. ${ }^{3}$ Only extensive lesions of the spinal cord produce postural hypotension because a decrease of $50 \%$ of preganglionic sympathetic neurons is necessary. ${ }^{2}$ Spinal cord diseases very rarely produce postural hypotension, which has been reported with cervical cord injuries, ${ }^{3}$ multisystem atrophy, ${ }^{4}$ advanced syringomyelia ${ }^{5}$ and multiple sclerosis. ${ }^{2}$ The higher the spinal cord injury the more severe is the fall of blood pressure. ${ }^{6}$ Postural hypotension following spinal cord injury is most pronounced in the acute stage, but later the patient develops a tolerance to upright posture, attributed to an increased sympathetic vasomotor reflex of the isolated spinal cord and an altered renal vascular renin receptor mechanism. ${ }^{7}$ Our patient developed a similar tolerance three months after the illness. Decompression sickness can also result in acute myelopathy which is attributed to occlusion of epidural veins by nitrogen bubbles leading to venus infarction of spinal cord. ${ }^{8}$ The MRI picture may reveal haemorrhagic changes similar to our patient. In syringomyelia, the involvement of intermediolateral horn cells and descending autonomic pathways have been reported to be responsible for postural hypotension. ${ }^{5}$ The progressive course, lack of compensation to orthostatic hypotension and characteristic MRI features would be distinctive.

Acute transverse myelitis generally results in segmental involvement of the thoracic spinal cord. Recent MRI studies have revealed a higher level of signal alteration, of more than six to 10 
segments above the sensory level. We also have reported similar results on the basis of MRI and central motor conduction studies. ${ }^{9}$ In view of our observation, postural hypotension of a milder degree may be commoner. Before initiating a rehabilitation programme in a patient with acute transverse myelitis, postural hypotension should be checked.

1 Barakos JA, Mark AS, Dillon WP, Norman D. MR imaging of acute transverse myelitis and AIDS myelopathy. $\mathcal{F}$ Comput Assist Tomogr 1990; 14: 45-50.

2 Zigler MG. Autonomic nervous system in the diseases of spinal cord. In: Davidoff RA, ed. Handbook of the spinal cord, Vol 4 \& 5 New York: Marcel-Dekker, 1987; pp 299-317.

Mathias CJ, Frankel HL. Clinical manifestation of malfunctioning sympathetic mechanism in tetraplegia. $\mathcal{f}$ Auton Nerv tioning sympathetic mech

4 Shy G, Drager G. A neurologic syndrome associated with orthostatic hypotension. Arch Neurol 1960; 2: 511-27.

5 Aminoff MJ, Wilcox CS. Autonomic dysfunction in syringomyelia. Postgrad Med f 1972; 48: 113-5.

\section{Learning point}

Postural hypotension, although rare, can occur in patients with extensive acute myelitis. It is

important to check for postural fall of blood

pressure before making the patient sit or stand

\section{Box 2}

6 Frankel HL, Mathias CJ. Cardiovascular aspects of autonomic dysreflexia since Guttmann and Witteridge (1947). Paraplegia 1979; 17: 46-51.

7 Alderson JD. Chronic care of spinal cord injury. In: Alderson JD and Frost EAM, eds. Spinal cord injuries. London: Butterworth, 1990; pp 104-25.

8 Hallenbeck JM, Bove AA, Elliott DH. Mechanism underlying spinal cord damage in decompression sickness. Neurology 1975; 25: 308-10.

9 Misra UK, Kalita J. Transverse myelitis - neurophysiological and MRI correlation. Paraplegia 1994; 32: 593-6. 